\begin{tabular}{|c|c|}
\hline Citation/Reference & $\begin{array}{l}\text { Carolina Varon, Jansen K., Lagae L., and Van Huffel S. (2015), } \\
\text { Can ECG monitoring identify seizures? } \\
\text { Journal of Electrocardiology, Accepted. }\end{array}$ \\
\hline Archived version & $\begin{array}{l}\text { Author manuscript: the content is identical to the content of the published } \\
\text { paper, but without the final typesetting by the publisher }\end{array}$ \\
\hline Published version & Not yet available \\
\hline Journal homepage & http://www.journals.elsevier.com/journal-of-electrocardiology/ \\
\hline Author contact & $\begin{array}{l}\underline{\text { carolina.varon@esat.kuleuven.be }} \\
+32(0) 16326417\end{array}$ \\
\hline IR & Not yet available \\
\hline
\end{tabular}

(article begins on next page) 


\section{Can ECG monitoring identify seizures?}

Carolina Varon ${ }^{\mathrm{a}, \mathrm{b}}$, Katrien Jansen ${ }^{\mathrm{c}}$, Lieven Lagae ${ }^{\mathrm{c}}$, Sabine Van Huffel ${ }^{\mathrm{a}, \mathrm{b}}$

${ }^{a}$ KU Leuven, Department of Electrical Engineering ESAT-STADIUS, Kasteelpark Arenberg 10, 3001 Leuven, Belgium. (email: carolina.varon, sabine.vanhuffel @esat.kuleuven.be)

biMinds Medical IT Department, Kasteelpark Arenberg 10, 3001 Leuven, Belgium

'Pediatric neurology, University Hospitals Leuven, UZ Herestraat 49, 3000 Leuven, Belgium. (email: katrien.jansen, lieven.lagae @uzleuven.be)

\section{Corresponding author}

Carolina Varon

Department of Electrical Engineering ESAT-STADIUS

KU Leuven

Kasteelpark Arenberg 10, 3001 Leuven, Belgium

tel. +3216326417

or +3216321709

e-mail: carolina.varon@esat.kuleuven.be 


\begin{abstract}
Background

Seizures affect the autonomic control of the heart rate and respiration, and changes in these two variables are known to occur during, and even before the EEG onset of the seizure.

Goal

This work aims to quantify these changes and use them to identify the ECG onset.

Methods

Single-lead ECG signals were recorded from patients suffering from focal and generalized seizures. Two algorithms are proposed, one quantifies changes in the QRS morphology using principal component analysis, and one assesses cardiorespiratory interactions using phase rectified signal averaging.

Results

Positive predictive values of $86.6 \%$ and $77.5 \%$ and sensitivities of $100 \%$ and $90 \%$ were achieved for focal and generalized seizures respectively.

\section{Conclusion}

Results for focal seizures are in accordance with the literature, and detection of generalized seizures is improved after including respiratory information.

Significance

These findings could improve monitoring systems in epilepsy, and closed-loop techniques that aim to stop seizures. 


\section{Highlights:}

- Cardiac and respiratory changes during epileptic seizures

- Cardiorespiratory interactions derived solely from the ECG signal

- Effect of epilepsy on cardiorespiratory interactions

- Cardiorespiratory information improves the detection of generalized seizures

Key words: epilepsy, respiration, autonomic, childhood, seizures 


\section{Introduction}

The control mechanisms of the autonomic nervous system are known to be deeply involved during epileptic seizures [1]. For example, changes in heart rate can occur during and even a few seconds before and after the EEG onset of the seizures $[2,3,4,5,6]$. These changes can be caused by either the involvement of the central autonomic centers during seizures, or by motor activity and/or stress responses to the seizures.

Different studies have shown that not only the heart rate, but also the morphology of the ECG changes during seizures (i.e. ictal activity). In [5] it was shown that the QT interval was shortened during the early post-ictal phase in patients suffering from refractory temporal lobe epilepsy. Additionally, ECG abnormalities such as T wave inversion and ST elevation/depression, have been observed in the preictal period of focal and generalized seizures [6]. These studies focused on the presence of ictal tachycardia in adults with refractory epilepsy. In [3] the focus was however, on childhood epilepsy and it was reported that changes in heart rate can be observed in temporal-lobe and frontal-lobe seizures. Generalized seizures present a bigger challenge due to deep motor involvement and/or a lack of significant changes in heart rate. The assumption made in $[2,3,4]$ was that seizures are characterized by a steady state phase followed by a significant increase (sometimes decrease) in heart rate that can be modelled using a quadratic function. This is of course valid in the presence of tachycardia or bradycardia, and when no ECG artifacts affect the detection of the R-peaks.

A different effect of epilepsy was observed in [7], where it was reported that epileptic seizures can be accompanied by apnea episodes, especially when the onset is localized in one of the temporal lobes. Moreover, as it was shown in [8], the cardiorespiratory interactions have a particular behavior towards the onset of focal and generalized seizures. Under these circumstances, it is possible to envision a detection system based on single-lead ECG that can improve the quality of life of patients suffering from epileptic seizures. This improvement in quality of life can be achieved due to the fact that the ECG has an inherent advantage over EEG in the sense that it is easier to record in a long-term ambulatory setting, hence, more portable systems can be developed. In addition, changes can be observed in the ECG even before the EEG onset, making it possible to early-detect the onset of a seizure. This last issue is very important for the development of "closed-loop" systems like vagus nerve stimulation, where the ultimate goal is to stop seizures from occurring and prevent sudden unexpected death in epilepsy (SUDEP) [9].

In order to contribute to the development of seizure detection systems based on more portable sensors, this paper presents a methodology that uses only the ECG signal to detect epileptic seizures. This methodology proposes a novel feature that describes the changes of the ECG morphology by means of 
principal component analysis (PCA). This new feature allows to identify changes of the morphology of the ECG, in particular of the QRS-complex, which can be caused by seizures. This study is the first one, where changes in the QRS morphology are quantified and used for the detection of epileptic seizures. Furthermore, this study uses phase rectified signal averaging (PRSA) and its bivariate version (BPRSA) to quantify changes in the cardiorespiratory interactions. These methodologies allow to quantify quasi-periodicities in both the heart rate, and in the way respiration triggers changes in the heart rate. In this study, it is hypothesized that during epileptic seizures, these quasi-periodicities will be affected due to sympathetic activations or due to the involvement of the cardiorespiratory control during the seizure.

Different types of seizures in childhood and adult epilepsy are studied, namely, focal seizures with onset in the frontal lobe or in one of the temporal lobes, and generalized seizures. The biggest challenge is to detect generalized seizures, especially those with deep motor involvement, and with no obvious effect in the heart rate.

\section{Methodology}

\section{Datasets}

Two datasets were used in this study. The first one consists of single-lead ECG recordings from children suffering from refractory epilepsy. These children were admitted to the epilepsy clinic of UZ Leuven in Belgium, and were monitored using 24-hour video-EEG-ECG. In total, 37 patients were monitored and 98 seizures were identified. The mean age of the patients was 9.2 years (range 3-16 years). The onset of the seizures was annotated based on EEG and video, by an EEG specialist. Two types of seizures were recorded: 48 with focal onset and 50 with generalized onset. Twenty of the focal seizures originated from the temporal lobe and the other 28 from the frontal lobe. The generalized seizures were 29 tonic or tonic-clonic, 11 myoclonic, and 10 generalized absence seizures. Once the seizures were identified, a 5 min ECG segment was extracted, starting 3 minutes before the EEG onset of the seizure. In this way, these ECG segments consist of 3 seizure-free or pre-ictal minutes and 2 post-ictal minutes. The ECG signals were recorded at a sampling frequency of $250 \mathrm{~Hz}$.

In order to test the algorithms on long term ECG recordings and on seizure-free segments longer than 3 minutes, the second dataset was used, namely the publicly available "Post-Ictal Heart Rate Oscillations in Partial(focal) Epilepsy" dataset [10]. This dataset contains 7 ECG recordings, extracted from video-EEG-ECG monitoring of 5 women whose age ranged from 31 to 48 years, and who were suffering from epilepsy. Ten focal seizures were recorded. The ECG signals were sampled at $200 \mathrm{~Hz}$, and their duration ranged from $1 \mathrm{~h} 30 \mathrm{~m}$ up to $3 \mathrm{~h} 45 \mathrm{~m}$. 
The R-peaks were detected from each dataset using the Pan-Tompkins algorithm. Ectopic and missing peaks were corrected using a search back procedure as in [8], and the respiratory information, or socalled ECG-derived respiration (EDR), was computed using the amplitude of the R-peaks as proposed in [11]. In the remaining of this paper, this derived respiratory signal will be denoted by $R_{a m p}$.

\section{Continuous quantification of ECG morphology changes}

After the R-peak detection, 5 signals were computed using the procedure described below, and outlined in Figure 1. This procedure was inspired by the derivation of the respiratory signal from the ECG using PCA [12].

a. A symmetric window of $120 \mathrm{~ms}$ ( $m$ samples) around each R-peak was selected in order to capture the QRS-complexes.

b. Five $(n=5)$ consecutive QRS complexes were assembled into one $Q R S$-matrix $Q \in \mathbb{R}^{\operatorname{mxm}}$, and aligned with respect to the R-peaks. The definition of $n$ was done experimentally, as the lowest amount of heart beats that lead to a higher positive predictive value (PPV).

c. Principal component analysis (PCA) was performed on $Q$, and the five non-zero eigenvalues were selected as the new set of parameters.

d. Shift one heart beat and repeat from step a, until the end of the ECG segment.

After this procedure, the evolution of the five non-zero eigenvalues was obtained with a resolution of 5 heart beats, and a vector of 5 features was used to characterize each window shift. This feature vector for the $i$ th window corresponds to $\left\{\lambda_{i, j}\right\}_{j=1}^{5}$.

Phase rectified signal averaging (PRSA)

The cardiorespiratory interactions were quantified using phase rectified signal averaging (PRSA), which is a methodology originally proposed in [13] to detect quasi-periodicities in non-stationary signals, in the presence of noise and artifacts. An example of such signals is the (resampled at $4 \mathrm{~Hz}$ ) RR interval time series $R R=\left\{r r_{i=1}^{N}\right\}$, with $N$ the total length of the signal, where several dynamic changes and interruptions in the periodic oscillations of the heart rate can be observed. The reason for this is related to the fact that the $R R$ signal is influenced and modulated by different physiological mechanisms at the same time. In order to deal with the non-stationary nature of $R R$, PRSA reduces the 
original signal of length $N, \boldsymbol{Y}=\left\{y_{i}\right\}_{i=1}^{N}$ into a compressed version $\boldsymbol{Y}^{s}=\left\{y_{j}^{\prime}\right\}_{j=-L}^{L}$, with $y_{n} y^{\prime} \in \mathbb{R}$, and $L \ll N$, where quasi-periodicities can be unveiled. Here, $N$ is the length of the ECG segment. The shorter sequence $\boldsymbol{Y}^{\prime}$ of length $2 L+1$ is computed following the three steps illustrated in Figure 2, and described next.

1. Anchor point (AP) selection: Given the time series $\boldsymbol{Y}$ all points $y_{i}$ that satisfy $y_{i}<y_{i-1}$ are selected as anchor points (AP). The AP correspond to accelerations of the heart rate.

2. Signal segmentation: A window of length $2 L+1$ is defined around each AP. When an AP is not surrounded by such a window, it is discarded from the analysis. In this way a total of $M$ AP and $M$ windows remain. $\mathrm{L}$ was set to 20 samples ( 5 seconds for $4 \mathrm{~Hz}$ ), which corresponds to the length that allows to see changes in coherence times within the signal.

3. Rectification and averaging: In this step all the windows are aligned around the AP. Then, $\boldsymbol{Y}^{\prime}$ or the so-called PRSA curve, is computed over all $M$ windows. This curve now contains information of quasi-periodicities related to decreases in the time series. All oscillations that are not phase synchronized with the AP are averaged out, as well as all the noise and artifacts.

In the bivariate version of PRSA, namely the BPRSA, steps 1 and 2 are performed in separate signals, which leads to the investigation of the interrelations between two different processes [14]. In BPRSA the AP selection is done on the "driver" signal $\boldsymbol{X}$ (i.e. respiration sampled at $4 \mathrm{~Hz}$ ), and the segmentation is performed on the "target" signal $\boldsymbol{Y}$ (i.e. heart rate). Once the target is segmented, the BPRSA curve is computed as the average between the segments. The right panels of Figure 2 indicate the computation of the BPRSA curve.

In order to simulate a continuous detection of epileptic seizures, PRSA was implemented using a moving window of 80 heart beats. The length of the window was determined experimentally, as the length that lead to a higher positive predictive value (PPV). Each pair of signals was then used to extract two different PRSA curves, one where $R R$ was used as trigger and target simultaneously, and one where the derived respiratory signal $R_{a m p}$ triggers the $R R$. This procedure was repeated for every window shift, in steps of one heart beat with an overlap of 79 beats (see Figure 2). 
Different parameters were used to quantify the (B)PRSA curves, and they correspond to the slopes of the lines connecting the point before and after the AP in the (B)PRSA curves. These features will be denoted by $S_{R R}$ and $S_{R}$ for PRSA and BPRSA, respectively. These slopes will give an indication on how fast the heart reacts to acceleration, and how the inspiration/expiration cycle is related to changes in the heart rate variability (HRV). As mentioned in the introduction, it is hypothesized that during epileptic seizures, these reaction times will be affected due to sympathetic activations or due to the involvement of the cardiorespiratory control during the seizure. Besides, it is expected that the coherence times within the signal change due to the significant changes in HRV that might take place in the pre-ictal/ictal period. The coherence time can be estimated from the slope of the line connecting points at a certain distance from the AP in the PRSA and BPRSA curves. These slopes will be denoted by $\Delta_{R R}=\left(Y^{\prime}{ }_{L}-Y^{\prime}{ }_{-L}\right) /(2 L+1)$ and $\Delta_{R}=\left(X^{\prime}{ }_{L}-X^{\prime}{ }_{-L}\right) /(2 L+1)$, respectively. Summarizing, for a moving window of 80 heart beats with an overlap of 79 beats and steps of 1 heartbeat, the $i$ th window was characterized by the following feature vector: $\left[S_{i, R R}, S_{i, R}, \Delta_{i, R R}, \Delta_{i, R}\right]$, with $i=80, \ldots, N$, and $N$ the total number of heart beats in the ECG segment.

In total, 11 features were extracted from the ECG, $R R$ and $R_{a m p}$, with a resolution of 1 heartbeat and they are summarized in Table 1 . The mean is defined as $\overline{R R_{l}}=1 / N \sum_{k=1}^{N} r r_{k}$, and the standard deviation $(\mathrm{SDNN})$ as $\sigma(R R)_{i}=\sqrt{1 / N \sum_{k=1}^{N}\left(r r_{k}-\overline{R R_{l}}\right)^{2}}$, for $N=5$ or $N=80$.

The methodology used to split the data into two different groups, normal and seizure, was kernel spectral clustering (KSC) [15]. This clustering algorithm was first trained using a subset of 405 minutes segments that were selected using the modified fixed-size algorithm proposed in [16]. Next, the 58 segments of 5-minutes and the Physionet dataset were used to test the algorithm. Since the seizure onset annotations were done on the EEG signal, and it is known that cardiac changes can be observed before or after these annotations, a symmetric window of 60 s around the annotated seizure onset was used to calculate the number of false positives. In other words, if the clustering algorithm produced a "seizure" result within 30s before or 30s after the annotated seizure onset, the result was considered a true positive. If on the other hand, the result was outside this 60 s window, the result was labelled as false positive.

\section{Results}

ECG morphology changes 
In the first approach, windows of 5 heart beats are analyzed and characterized by:

$\left[\overline{R R}_{i}, \sigma(R R)_{i}, \lambda_{i, 1}, \lambda_{i, 2}, \lambda_{i, 3}, \lambda_{i, 4}, \lambda_{i, 5}\right]$. The clustering algorithm KSC was trained using all windows in the 5-minute segments containing 40 seizures: 20 focal and 20 generalized. Each cluster was then manually characterized as containing seizure or normal activity, and test points were associated to either cluster using the out-of-sample extensions of KSC proposed in [15]. It was observed that changes in the ECG morphology around the onset of the seizure are more visible in $\left[\lambda_{i, 3}, \lambda_{i, 4}, \lambda_{i, 5}\right]$. The reason for this is that the first two components are more affected by the respiratory modulation [12], and the other components become more relevant when the morphology of the QRS complex becomes more heterogeneous. Therefore, the feature set used at this point was $\left[\overline{R R}_{i}, \lambda_{i, 3}, \lambda_{i, 4}, \lambda_{i, 5}\right]$. In total, $85 \%$ of the focal seizures, and only $57 \%$ of the generalized seizures were detected (see Table 2 ) using the segments of 5 minutes. Then, the Physionet dataset was used to test the algorithm, and a PPV of $52.6 \%$ was achieved for long-term ECG signals.

Table 1. Set of features derived from the ECG, $R R$-interval time series, and respiratory signal $R_{\text {amp }}$. The parameters are computed for each window of 5 heart beats for the morphology approach, and of 80 heart beats for the phase rectified signal averaging (PRSA) approaches.

\begin{tabular}{|c|c|c|c|c|}
\hline \multirow{2}{*}{ Method } & Feature ID & \multicolumn{3}{|c|}{ Derived from } \\
\cline { 2 - 4 } & \multicolumn{2}{|c|}{ ECG } & RR & Ramp \\
\hline Mean* & $\sigma(R R)_{i}$ & $\mathrm{X}$ & \\
SDNN* & {$\left[\lambda_{i, 1}, \lambda_{i, 2}, \lambda_{i, 3}, \lambda_{i, 4}, \lambda_{i, 5}\right]$} & $\mathrm{X}$ & $\mathrm{X}$ & \\
Morphology & {$\left[S_{i, R R}, \Delta_{i, R R}\right]$} & & $\mathrm{X}$ & \\
PRSA & {$\left[S_{i, R}, \Delta_{i, R}\right]$} & & $\mathrm{X}$ & $\mathrm{X}$ \\
BPRSA & & & & \\
\hline
\end{tabular}

* These parameters are calculated for each moving window of 5 or 80 heart beats.

About $75 \%$ of the changes in the ECG were lagging the onset in the EEG, while the rest was leading or simultaneous. The detection window for focal seizures was $7.4 \pm 14.3$ seconds, and for generalized $9.6 \pm 20.5$ seconds. Keep in mind that some of the generalized seizures used in this study involve motor activity, which implies the presence of movement artifacts on the ECG that reduce the reliability of the R-peak detection, and seizure detection algorithms. For this reason, the ECG should be cleaned from artifacts, and different features derived from signals like the electromyogram (EMG) and/or accelerometers should be used to complement this study. In this work, the short-term ECG segments (5 minutes) were manually verified for erroneous R-peak detections. On the other hand, the long-term ECG signals of the Physionet dataset were first pre-processed using the artifact detection algorithm 
proposed in [17], where contaminated segments were removed from the analysis, and then the R-peaks were detected without further visual inspection.

\section{Phase rectified signal averaging}

In this approach, windows of 80 heart beats were used, and the set of features that showed clear changes around the seizure onset corresponds to $\left[S_{i, R R}, \Delta_{i, R R}, \Delta_{i, R}\right]$. The same training and test sets selected in the previous approach were used here, and the total results are indicated in Table 2. In the dataset of 5-minutes segments, 9 out of the 11 myoclonic seizures were detected but 6 false positives were also included (PPV=60\%). If these seizures are excluded from the generalized group, the total PPV was $83.7 \%$. On the other hand, if the seizure segments contaminated by EMG, namely 24 seizures (48\%), are removed from the set, the PPV increases to $89 \%$ and all the generalized seizures are detected. Again, other modalities like EMG or accelerometer should be included in the analysis of motor seizures. The results obtained using (B)PRSA were compared with those reported using the previous approach (i.e. morphology of the ECG), but in this case, an improved PPV was achieved for both types of seizures, and a reduction of more than 50\% was achieved in the amount of false positives for the generalized seizures compared with the previous approach. For long-term ECG signals on the other hand, a PPV of 71.4\% was reached. Note that the KSC model was trained on 40 segments of 5minutes recorded in the UZ Leuven, and still the algorithm was capable to detect all focal seizures in the Physionet dataset, and produce a relatively high PPV. A total PPV for focal seizures, including both the 5-minute set and the Physionet dataset is $86.6 \%$, with sensitivity of $100 \%$.

Concerning the latency of detection, it was found that with (B)PRSA, $81 \%$ of the seizures were detected more than 20 seconds ( $24.8 \pm 3.9$ seconds) after the seizure onset in the EEG. These delays are caused by the fact that a window of 80 heart beats is used in this approach.

Table 2 Seizure detection results

\begin{tabular}{|l|c|c|c|c|c|}
\hline \multicolumn{1}{|c|}{ Seizures } & Algorithm & TP & FP & PPV & Sens \\
\hline \multirow{2}{*}{ Focal } & Morphology & 43 & 7 & $85.7 \%$ & $89.5 \%$ \\
& (B)PRSA & $\mathbf{4 8}$ & $\mathbf{5}$ & $\mathbf{9 0 . 5 \%}$ & $\mathbf{1 0 0 \%}$ \\
\hline \multirow{2}{*}{ Generalized } & Morphology & 43 & 32 & $57.3 \%$ & $86 \%$ \\
& (B)PRSA & $\mathbf{4 5}$ & $\mathbf{1 3}$ & $\mathbf{7 7 . 5 \%}$ & $\mathbf{9 0 \%}$ \\
\hline $\begin{array}{l}\text { Physionet } \\
\text { (Focal) }\end{array}$ & Morphology & 10 & 9 & $52.6 \%$ & $100 \%$ \\
\hline
\end{tabular}




\section{Discussion}

These results are in accordance with previous studies [2] where ECG segments of 2 minutes were analyzed, and a PPV larger than $80 \%$ for focal seizures was achieved. The methodologies used in [2, 3] studied the full ECG segment of few minutes in order to find the best curve fitting, and the exact seizure onset. In other words, the detection can be done only offline. The methodologies proposed in this paper use a moving window of 5 and 80 heartbeats, hence, the detection of the seizure could be done in an online fashion, with a time delay of up to 30 seconds. In the case of the analysis using a window of 80 heartbeats, it is worth noting that in order to detect the epileptic seizure, up to 30 seconds of the seizure activity need to be contained within the window. In other words, the first seconds of the window might contain seizure-free activity, while the most recent heartbeats should belong to the seizure.

Only the detection of focal seizures was evaluated in a long-term setting, while the results of generalized seizures were obtained using selected segments of 5 minutes around the seizure onset. This last point reduces the applicability of the proposed algorithms in the online detection of generalized seizures.

It is not a surprise that the results for generalized seizures are affected by myoclonic seizures, since they are brief, normally lasting no more than 2 seconds, and more importantly, they manifest themselves as rapid contraction/relaxation of a muscle. This implies motor involvement and very short changes $(<2 s)$. For this reason, the detection of these seizures remains a challenge. Nevertheless, it was shown that by including respiratory information as in BPRSA, the results for this type of seizures were improved. These findings indicate that seizures affect not only the heart rate but also its interactions with respiration.

When both proposed methodologies are compared, it is clear that a compromise must be made between number of FP and latency of the detection. This will always depend on the application. For instance, for seizure detection, where it is required to detect seizures as soon as possible, the algorithm based on morphology can be considered. On the other hand, for diagnosis or follow-up of therapies an algorithm like the one based on (B)PRSA that has a higher latency of detection but a lower false positive rate can be considered. Keep in mind however, that the performance of the proposed algorithms is significantly reduced by ECG contaminants, like movement artifacts. Therefore, other modalities must be used to achieve a high performance in the detection of seizures with motor involvement, and in ambulatory systems where movement artifacts are unavoidable.

It is worth noting that the results presented in this study indicate that a single-lead ECG signal can be used to detect epileptic seizures with a relatively high PPV. In addition, not only heart rate information was used, but also the respiratory effort was derived from the morphology changes of the ECG. As a 
result, it is possible to consider monitoring systems for epilepsy based solely on the ECG. This will reduce costs and increase comfort in ambulatory systems.

\section{Conclusion}

This paper presented two different algorithms for the detection of epileptic seizures from a single-lead ECG. The first one captured changes in the morphology of the QRS complexes by means of principal component analysis, and the second one quantified using (B)PRSA, the acceleration of the heart rate caused by an epileptic seizure. The algorithms were trained using ECG segments of 5 minutes, and tested on similar segments and on long-term ECG signals. Both algorithms detected focal epileptic seizures with a PPV of about $80 \%$, in a window of 30s around the EEG onset. For generalized seizures, however, the performance went down, in particular when myoclonic seizures were included. Nevertheless, the approach based on PRSA allowed to detect generalized seizures with a PPV of 83\%. Consequently, it is necessary to keep in mind that the ECG needs to be cleaned from EMG and movement artefacts in order to be able to detect seizures with motor involvement, as it is the case for the generalized seizures used in this work and some focal seizures involving the frontal lobe.

A clear disadvantage of the proposed algorithms is that an accurate R-peak detection is required. Hence, any ECG contaminant would reduce the performance of the algorithms, therefore, they must be detected and considered in the detection algorithm. Furthermore, the results obtained for generalized seizures were only achieved for segments of 5 minutes, which is a limitation of this study. Therefore, further analysis of these seizures must be performed in long-term ECG signals in order to draw stronger conclusions.

The proposed algorithms are easy to implement and can be adapted to home monitoring or online detection systems. However, training and validating the algorithm must be considered for each type of seizures, and different sources of data should be included to improve the performance for seizures with motor involvement. In other words, better performances can be expected if these algorithms are used to complement other approaches such as those based on accelerometers and EMG. In addition to other modalities, future research should also focus on merging both methodologies in long-term ECG recordings.

\section{Acknowledgements}

This research was supported by: Bijzonder Onderzoeksfonds KU Leuven (BOF) Center of Excellence (CoE) \#: PFV/10/002 (OPTEC). Fonds voor Wetenschappelijk Onderzoek-Vlaanderen (FWO) Project \#: G.0427.10N (Integrated EEG-fMRI); G.0108.11 (Compressed Sensing); G.0869.12N (Tumor imaging); G.0A5513N (Deep brain stimulation). Agentschap voor Innovatie door Wetenschap en Technologie (IWT) Project \#: TBM 080658-MRI (EEG-fMRI); TBM 110697-NeoGuard. iMinds Medical Information Technologies: Dotatie-Strategisch basis onderzoek (SBO- 2015); ICON: NXT_Sleep. Belgian Federal Science Policy Office: IUAP \#P7/19/ (DYSCO, `Dynamical systems, control and optimization', 2012-2017). Belgian Foreign Affairs-Development Cooperation: VLIR UOS programs (2013-2019). EU: European Union's Seventh Framework Programme (FP7/2007- 
2013): EU MC ITN TRANSACT 2012, \#316679; ERASMUS EQR: Community service engineer, \#539642-LLP-1-2013. Other EU: INTERREG IVB NWE programme \#RECAP 209G. European Research Council: ERC Advanced Grant, \#339804 BIOTENSORS This paper reflects only the authors' views and the Union is not liable for any use that may be made of the contained information.

\section{References}

[1] L. Blumhardt, P. Smith en L. Owen, „Electrocardiographic accompaniments of temporal lobe epileptic seizures," The Lancet, vol. 327, nr. 8489, pp. 1051--1056, May 1986.

[2] F. Leutmezer, C. Schernthaner, S. Lurger, K. Ptzelberger en C. Baumgartner, „Electrocardiographic changes at the onset of epileptic seizures," Epilepsia, vol. 44, pp. 348-354, 2003.

[3] K. Jansen, C. Varon, S. Van Huffel en L. Lagae, „Peri-ictal ECG changes in childhood epilepsy: Implications for detection systems," Epilepsy \& Behavior, vol. 29, pp. 72-76, 2013.

[4] W. J. Van Elmpt, T. M. Nijsen, P. A. Griep en J. B. Arends, „A model of heart rate changes to detect seizures in severe epilepsy," Seizure, vol. 15, nr. 6, pp. 366--375, 2006.

[5] R. Surges, C. A. Scott en M. C. Walker, „Enhanced QT shortening and persistent tachycardia after generalized seizures," Neurology, vol. 74, nr. 5, pp. 421--426, 2010.

[6] M. Zijlmans, D. Flanagan en J. Gotman, „Heart rate changes and ECG abnormalities during epileptic seizures: prevalence and definition of an objective clinical sign," Epilepsia, vol. 43, nr. 8, pp. 847-854, 2002.

[7] M. E. O'Regan en J. K. Brown, „Abnormalities in cardiac and respiratory function observed during seizures in childhood," Developmental Medicine \& Child Neurology, vol. 47, nr. 1, pp. 4-9, 2005.

[8] C. Varon, K. Jansen, L. Lagae, L. Faes en S. Van Huffel, „Transient behavior of cardiorespiratory interactions towards the onset of epileptic seizures," in Proceedings of the 41st annual computing in cardiology (CinC), Boston, 2014.

[9] A. Van de Vel, K. Cuppens, B. Bonroy, M. Milosevic, K. Jansen, S. Van Huffel, B. Vanrumste, L. Lagae en B. Ceulemans, „Non-EEG seizure-detection systems and potential SUDEP prevention: state of the art," Seizure, vol. 22, nr. 5, pp. 345--355, 2013.

[10] I. Al-Aweel, K. Krishnamurthy, J. Hausdorff, J. Mietus, J. Ives, A. Blum, D. Schomer en A. Goldberger, „Postictal heart rate oscillations in partial epilepsy,” Neurology, vol. 53, nr. 7, pp. 1590-1590, 1999.

[11] G. B. Moody, R. G. Mark, A. Zoccola en S. Mantero, „Derivation of respiratory signals from multilead ECGs," Computers in cardiology, vol. 12, pp. 113--116, 1985.

[12] P. Langley, E. J. Bowers en A. Murray, „Principal component analysis as a tool for analyzing beatto-beat changes in ECG features: application to \{ECG\}-derived respiration," IEEE Transactions on Biomedical Engineering, vol. 57, nr. 4, pp. 821--829, 2010.

[13] A. Bauer, J. W. Kantelhardt, A. Bunde, P. Barthel, R. Schneider, M. Malik en G. Schmidt, „Phaserectified signal averaging detects quasi-periodicities in non-stationary data," Physica A: Statistical Mechanics and its Applications, vol. 364, pp. 423--434, 2006. 
[14] A. Y. Schumann, J. W. Kantelhardt, A. Bauer en G. Schmidt, „Bivariate phase-rectified signal averaging," Physica A: Statistical Mechanics and its Applications, vol. 387, nr. 21, pp. 5091--5100, 2008.

[15] C. Alzate en J. A. Suykens, „Multiway spectral clustering with out-of-sample extensions through weighted kernel PCA," IEEE Transactions on Pattern Analysis and Machine Intelligence, vol. 32, nr. 2, pp. 335-347, 2010.

[16] C. Varon, C. Alzate en J. Suykens, „Noise level estimation for model selection in kernel PCA denoising," IEEE transactions on neural networks and learning systems (in press), 2015.

[17] C. Varon, D. Testelmans, B. Buyse, J. Suykens en S. Van Huffel, „Robust artefact detection in long-term ECG recordings based on autocorrelation function similarity and percentile analysis," in the 34th annual international conference of the IEEE Engineering in Medicine and Biology Society, San Diego, California, 2012.

\section{$\underline{\text { Figures }}$}

a)

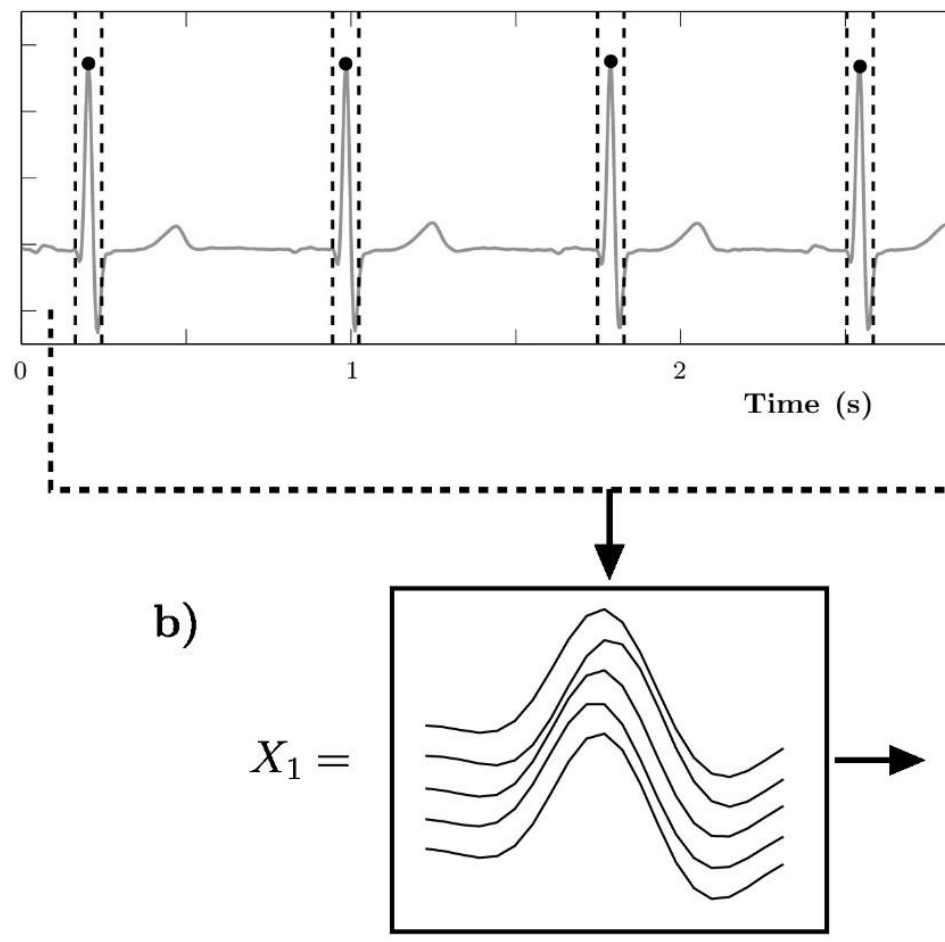

c)

$$
\begin{aligned}
\Sigma_{1} & =X_{1} X_{1}^{T} \\
\Sigma_{1} \alpha_{1}^{(j)} & =\lambda_{1, j} \alpha_{1}^{(j)}
\end{aligned}
$$

Time (s)

Figure 1. Procedure to construct the QRS-matrix, and derive the five non-zero eigenvalues $\lambda_{1_{1 j} j}$ of the matrix $Q_{1}$, where $j=1_{x}, 5$. The window of 5 heart beats is shifted throughout the whole ECG segment and the evolution of the eigenvalues $\lambda_{i_{j} j}$ is computed, where $i=1_{w a x} N$ and $N$ the number of heart beats in the segment. 

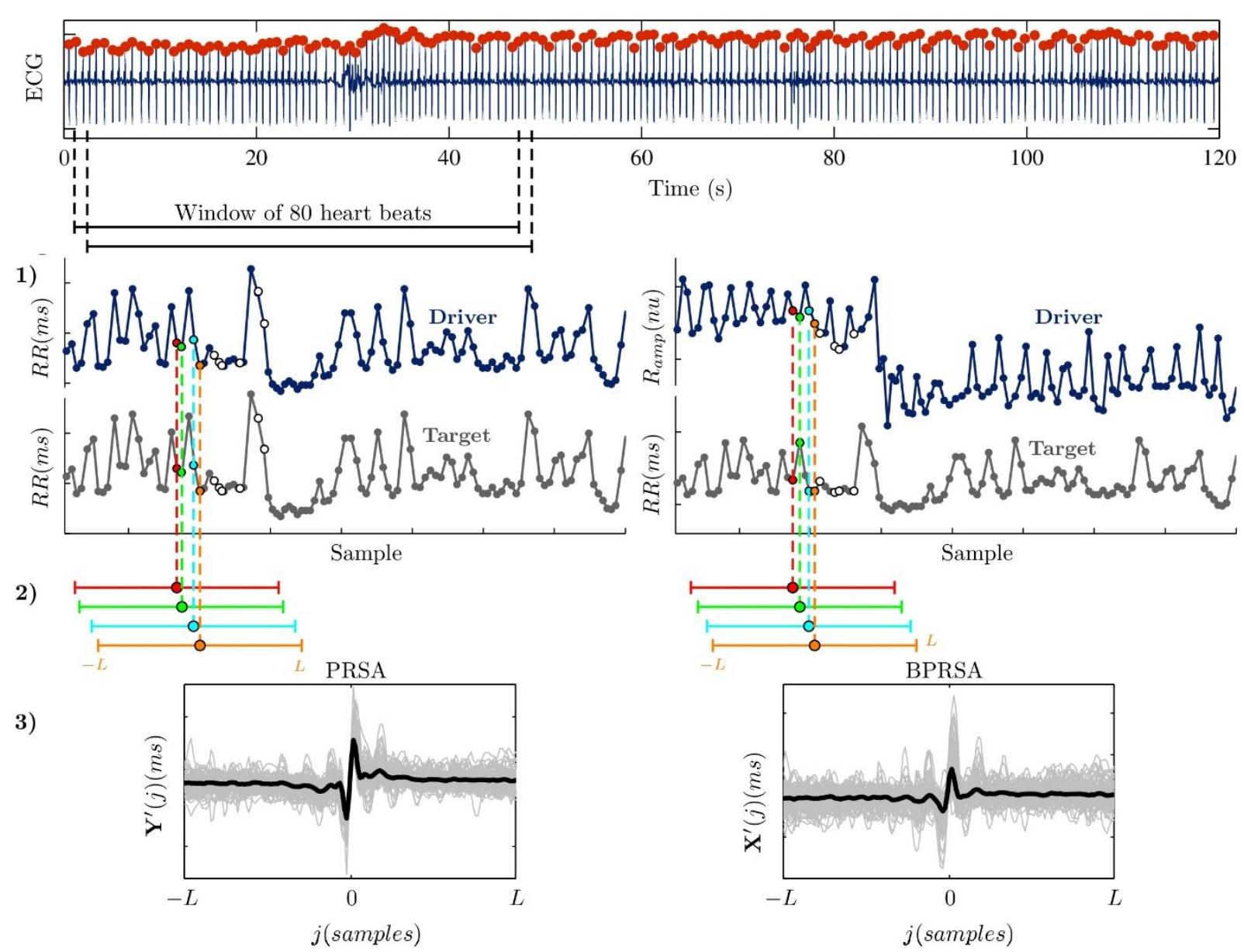

Figure 2. Illustration of the computation of the (B)PRSA curves. A window of 80 heart beats in the ECG is used for the illustration, and from each window, two signals are computed: $R R$ and $R_{a m p}$. 1) Selection of anchor points in the driver signal $R R$ (left) and $\boldsymbol{R}_{\text {amp }}($ right). The first 12 AP are indicated. 2) Segmentation of the target signal $R R$, using a window of length $2 L$ around each anchor point. 3) Phase rectification and signal averaging of all extracted segments around AP. The average (B)PRSA curves are indicated in black. nu stands for normalized units. 